Check for updates

Cite this: RSC Adv., 2018, 8, 29062

\section{Thermal dewetting tunes surface enhanced resonance Raman scattering (SERRS) performance}

\author{
Sonia Andrikaki, ${ }^{\text {ab }}$ Katerina Govatsi, ${ }^{\text {ac }}$ Spyros N. Yannopoulos, (D) a \\ George A. Voyiatzis (iD ${ }^{a}$ and Konstantinos S. Andrikopoulos (iD *a
}

\begin{abstract}
Surface Enhanced Raman Spectroscopy (SERS) belongs to the techniques of ultra-sensitive chemical analysis and involves both identification and quantification of molecular species. Despite the fact that theoretically derived enhancement factors imply that even single molecules may be identified, which in some cases has indeed been experimentally observed, the application of this specific technique as an analytical tool is still an open field of research due to the need for reproducible, stable and simple to prepare SERS active substrates. The current work attempts to contribute to the already established knowledge on the substrates of metallic nanostructured films by a systematic study on the optimal conditions required for the detection of a specifically selected (model) material, the antitumor drug mitoxantrone (MTX). Au thin film deposition on Si substrates, by sputtering followed by solid state thermal dewetting is a facile and reproducible way to prepare Au nanoparticles with the desired particle size distribution. This offers control over their optical - plasmon resonance - properties that can be efficiently tailored to the prerequisites of the resonance Raman conditions, associated to the species under inspection, which is a supplement to the overall enhancement scattering factor. Furthermore, this work attempts to confirm the quantification capabilities of SERS, via the aforementioned substrates, in

view of extending SERS applications to food safety, biosensors etc.
\end{abstract}

Received 25th June 2018 Accepted 6th August 2018

DOI: $10.1039 / \mathrm{c} 8 \mathrm{ra05451 \textrm {g }}$

rsc.li/rsc-advances

\section{Introduction}

More than 80 years have already passed since the discovery of the Raman effect and Raman spectroscopy has become one of the most important analytical methods that enable structural characterization of materials at a molecular level. One of the most interesting and significant findings in this field is undoubtedly surface-enhanced Raman scattering (SERS) which was discovered in 1977 . According to this phenomenon, molecules adsorbed onto metal nanostructured surfaces, under certain conditions, may exhibit an anomalously large Raman cross section. ${ }^{1}$ This offers the possibility to identify molecular species at extremely low concentrations and it is anticipated that quantitative measurements could be achieved in the same low concentration levels. Among the most critical parameters for SERS is the active substrate. Colloidal SERS substrates are advantageous with respect to their easy preparation, low cost and concurrent high Raman enhancement factors. ${ }^{2}$ In comparison, solid substrates enable flexibility in sampling. ${ }^{3}$ A major handicap of colloidal substrates is their

\footnotetext{
${ }^{a}$ Foundation for Research and Technology, Hellas-Institute of Chemical Engineering Sciences (FORTH/ICE-HT), PO Box 1414, Rio-Patras, GR-26504, Greece. E-mail: candrik@iceht.forth.gr

${ }^{b}$ Department of Materials Science, University of Patras, Rio-Patras, GR-26500, Greece ${ }^{c}$ Department of Chemistry, University of Patras, Rio-Patras, GR-26500, Greece
}

low reproducibility with respect to SERS intensities. Thus, different colloidal batches may give different SERS intensities despite their similar preparation method, because hot-spots are not easy to be probed. In order to overcome this issue, flow $^{4}$ as well as oscillating ${ }^{5}$ cells have been proposed. Moreover, colloidal stability over long timescales is an additional negative factor for the reproducibility of quantification measurements. Solid substrates have been introduced over the past years targeting mainly to the detection of molecular species at extremely low concentrations. To this end, a number of inventive nanostructures have already been proposed. Among the simplest SERS solid substrates are thin metallic films applied usually on flat surfaces (e.g. $\mathrm{SiO}_{2}$, Si etc.). Gold/ silver coated flat surfaces have been studied for quite a long time with respect to their SERS efficiency. Several issues have already been concerned such as the substrate type in connection to the molecular species to be probed, the quantity of the metallic thin layer (typically referred as the nominal thickness on the substrate), the dewetting parameters (temperature and time), excitation parameters (wavelength and power). For practical purposes (including bionanosensors etc.) simple, reproducible and cost effective substrates are the most favourable. The morphology of these developed metallic nanostructures has been the subject of a number of research works which used several characterization techniques such as Scanning Electron Microscopy (SEM), 
Transmission Electron Microscopy (TEM), Scanning Tunneling Microscopy (STM) and Atomic Force Microscopy (AFM). Schlegel and Cotton, ${ }^{6}$ using TEM, studied the relationship of the deposition rate of thin silver films with SERS intensities and showed that slowly deposited films produce higher SERS intensities than rapidly deposited ones. Pavaskar et $a .^{7}$ studied the $\mathrm{Au}$ and $\mathrm{Ag}$ evaporated ultrathin films ( $\sim 5$ $\mathrm{nm}$ ) and using Finite-Difference Time Domain (FDTD) simulations, calculated the SERS enhancement factors. They verified that macroscopically observed SERS is dominated by a few number of nm-sized "hot spots" (distributed over large areas) whose enhancement factor reaches $10^{8}$. As such, these films utilize only a very small fraction of the sample area which strongly supports the concept that if only these films had greater number of "hot spots", much higher enhancement factors over the whole film could be obtained. In the same context, they found that (a) the Au films exhibit higher electric field intensities than $\mathrm{Ag}$, due to the smaller gaps between the existing islands and (b) the thermal treatment on the same thin films had a suppressing effect on the enhancement factors.

One of the earliest meticulous studies on quantification measurements through SERS using metallic island substrates was the one of R. P. Van Duyne et al., ${ }^{8}$ where the 3D morphological features of the SERS active pre-roughened/smooth substrates was revealed by AFM. Temperature besides initial film thickness was taken into consideration and the collected data were interpreted in the framework of the electromagnetic theory of SERS.

The deposition variables such as film thickness, evaporation rate and substrate temperature have been also studied ${ }^{9}$ for both metals, Au and Ag, by SERS effect. An increase in film thickness and low evaporation rates were shown to upshift the wavelength at maximum optical density $\left(\lambda_{\max }\right)$ and increase the optical density of the substrates. In contrast, pre- and postdeposition annealing of gold films led to the formation of substrates that exhibited a downshift of $\lambda_{\max }$.

The morphological parameters, monitored in situ and in real-time, of Ag nanostructured thin films deposited at ambient temperature over macroscopic areas on Si substrates and their effect on SERS signal have been studied. ${ }^{10}$ Maximum enhancement for the case of thiophenol was observed at the Ag percolation threshold which enabled to probe concentrations of $10^{-10} \mathrm{M}$ corresponded to enhancements of roughly 7 orders of magnitude.

A recent work ${ }^{\mathbf{1 1}}$ uses gold arrays on glass substrates and examines the influence of thickness, annealing temperature and excitation power; the rhodamine 6G SERS signal was found to increase by a factor of ten when thermal treatment (at $250{ }^{\circ} \mathrm{C}$ ) was applied on the gold arrays (10 $\mathrm{nm}$ nominal thickness).

Temperature has been also found to play role on the SERS intensities collected from films of Au nanoparticles prepared by the seed/growth method. ${ }^{\mathbf{1 2}}$

Particle sizes of 120-135 $\mathrm{nm}$ for the case of Au and Pd coated $\mathrm{Au}$ nanoparticle films were found to exhibit the highest SERS activity with the $632.8 \mathrm{~nm}$ excitation, which is much larger than that reported by the previous works, and is supported by FDTD

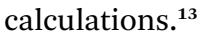

A correlation between dewetting temperature and SERS intensities offered by $\mathrm{Ag}$ nano-islands was thoroughly investigated experimentally and by simulations in a very recent work. ${ }^{\mathbf{1 4}}$ The authors conclude that the temperature dependence of the SERS intensity exhibits a characteristic maximum at $450{ }^{\circ} \mathrm{C}$ when using the specific temperature rates during their experiments.

In the current work, the morphological and optical properties of ultrathin Au-island films on Si substrates are investigated with the intention to highlight their capabilities as low cost optical sensors for applications requiring both identification and quantification of chemical species at low concentrations.

Methodologies are being proposed regarding the optimization of the collected Raman intensity by taking advantage of both the plasmonic properties control through the metal dewetting processes and the resonance Raman phenomenon associated with the particular molecular species requiring detection. Additionally, quantification capabilities are revealed.

The SERS substrates selected are composed of thermally dewetted Au coated Si wafers. The prototype molecular species demonstrating the above mentioned targets is mitoxantrone (MTX), which is a synthetic chemotherapeutic agent with proven antitumor activity against acute leukemia, ${ }^{15}$ breast cancer, ${ }^{16}$ non-Hodgkin's lymphoma ${ }^{17}$ and ovarian cancer. ${ }^{18}$ The tuning of plasmonic action by controlled dewetting with the aim of achieving resonance Raman Effect as an additional enhancement factor to SERS may as well be applied to other types of substrates besides Si wafers; furthermore, it may as well apply to identification and quantification of different molecular species besides MTX.

The detection of MTX using thick Au films as SERS and fluorescence substrates has been previously reported by Strekal et al. ${ }^{19}$ The authors found that the spectrum of MTX obtained from non-annealed thick Au films is similar to the one from $\mathrm{Ag}$ colloids. However, annealing at $240{ }^{\circ} \mathrm{C}$ led to the disappearance of SERS spectrum and an enhancement of fluorescence, while annealing at $340{ }^{\circ} \mathrm{C}$ led to the fluorescence quenching and a detection of a strong Raman spectrum. The work gave emphasis mostly on the parallel SERS and fluorescence spectral recording showing less effort on the substrate characterization of the as prepared and annealed films (by AFM). Here we attempt a systematic study of the correlation of surface morphology and SERS activity of MTX.

In this context, thin $\mathrm{Au}$ films of various thicknesses $(<5 \mathrm{~nm})$ were deposited by sputtering onto Si wafers $\langle 100\rangle$. The solid state thermal dewetting of Au films at various temperatures and for different time intervals resulted in the formation of $\mathrm{Au}$ nanoparticles. The initial film thickness controls the resulting particle size distribution. ${ }^{20}$ The optical properties of the substrates were studied by UV-Vis spectroscopy and their enhancement on the Raman signal was evaluated by observing the Raman features of the active agent MTX that was properly spin coated on the substrates. Correlation of SERS signal with the optical properties as well as the mean particle size of $\mathrm{Au}$ 
was attempted and optimum conditions for maximum SERS signal were investigated.

\section{Experimental}

\subsection{Materials and substrates}

Si wafers $\langle 100\rangle$ (Ted Pella Inc.) served as substrates. Prior to $\mathrm{Au}$ deposition, the Si chips $\left(1 \times 1 \mathrm{~cm}^{2}\right)$ were thoroughly cleaned by bath sonication in ethanol. Au films of various nominal thicknesses in the range of 1 to $5 \mathrm{~nm}$ were deposited using the low vacuum $\left(5 \times 10^{-2} \mathrm{mbar}\right)$ sputtering technique. The samples were annealed at two different temperatures $\left(400\right.$ and $\left.900{ }^{\circ} \mathrm{C}\right)$ for various time intervals. The selected parameters concerning the annealing conditions (time and temperature) and the nominal Au thickness played a decisive role in the morphology of the films regarding the average size and shape of the islands formed as well as the average distance between them.

Mitoxantrone dihydrochloride (MTX) was purchased from Sigma-Aldrich. Aqueous (triple distilled water) solutions of MTX at several concentrations $\left(0.5 \mu \mathrm{g} \mathrm{ml}^{-1}, 1 \mu \mathrm{g} \mathrm{ml}^{-1}, 1.5 \mu \mathrm{g} \mathrm{ml}^{-1}, 2\right.$ $\mu \mathrm{g} \mathrm{ml}{ }^{-1}, 10 \mu \mathrm{g} \mathrm{ml}^{-1}$ ) were prepared. In order to study the SERS response of the $\mathrm{Au}$ coated $\mathrm{Si}$ wafers the MTX molecules were dispersed on the substrates by spin coating small quantities ( 20 $\mu \mathrm{l})$ of the previously mentioned aqueous solutions.

\subsection{Characterization techniques}

The morphology of the Au coated Si wafers was studied by scanning electron microscopy (SEM) - Zeiss SUPRA 35 VP-FEG equipped with EDS (Bruker $\mathrm{GmbH}$, Quanta200) and BSE detectors (KE Developments, Ltd), operating at 5-20 keV.

The plasmon resonances of the Au islands on each substrate were detected by UV-Vis spectroscopy [Hitachi U-3000 reverse optics spectrophotometer] operating at specular reflection mode.

For the collection of the Raman spectra the excitation beams of a He-Ne laser (Optronics Technologies S.A. model HLA-20P, $20 \mathrm{~mW}$ ), an Ar+ laser (Stabilite 2017, SpectraPhysics) and a diode laser (Crystal Laser, RCL-080-785S) operating at $632.8,514.5$ and $785 \mathrm{~nm}$ wavelengths, respectively, were alternatively used. The laser power on sample was kept at $\sim 0.6$ $\mathrm{mW}$ and was focused using a long working distance $50 \times(\mathrm{NA}=$ 0.55) microscope objective. The scattered radiation was collected and analyzed by the T-64000 Jobin Yvon (ISA-Horiba group) $\mu$ Raman system using its single spectrograph configuration. The rejection of the elastic scattered photons was accomplished by appropriate edge filters. The Raman photons were dispersed by a 600 grooves per $\mathrm{mm}(76 \mathrm{~mm} \times 76 \mathrm{~mm})$ grating and detected by a standard $\mathrm{LN}_{2}$ cooled, at $140 \mathrm{~K}$ (Symphony II by Horiba 2D CCD, 1024X256). For statistical reasons a set of 30 spectra was collected from various positions on each sample's surface. Amounts of the spin coated material were visually inspected at the edges of the substrates. Spectral accumulation from these regions resulted in huge fluorescent background (when excited with $632.8 \mathrm{~nm}$ ) and since it did not contribute to the quantification measurements was deliberately avoided. Moreover, this particular portion of the material was carefully and thoroughly removed using a cotton swab under a stereomicroscope. For each substrate the intensity of a characteristic MTX Raman band was monitored as a function of nominal MTX concentration in order to verify its linearity and thus establish semi-quantitative results. Actual quantification requires more elaborate experiments (as described below) since the concentration of MTX on each of the studied surface is not known.

\subsection{Quantification of MTX}

An attempt to achieve quantification results and in consequence estimate the enhancements attained was performed by trying to apply a more concrete quantification procedure for the case of MTX spin coated on the substrates. The substrates were immerged in triple distilled water within a beaker put under sonication conditions for $15 \mathrm{~min}$. Quantification was then performed by standard quantification procedures for liquid samples using $\mathrm{Ag}$ colloids and $90^{\circ}$ scattering (macroRaman). ${ }^{2}$

\section{Results and discussion}

A typical Surface Enhanced Resonance Raman Scattering (SERRS) spectrum of MTX collected using the $632.8 \mathrm{~nm}$ excitation wavelength (i.e. in resonance Raman conditions according to the absorption spectrum in the inset) at $90^{\circ}$ scattering geometry from an Ag colloidal substrate is shown in Fig. 1. The molecular structure of MTX $\cdot 2 \mathrm{HCl}, \mathrm{C}_{22} \mathrm{H}_{28} \mathrm{~N}_{4} \mathrm{O}_{6} \cdot 2 \mathrm{HCl},\left(517.40 \mathrm{~g} \mathrm{~mol}^{-1}\right)$ is also shown as an inset. As has already been reported, the vibrational modes of MTX aqueous solutions using conventional Raman (632.8 nm excitation wavelength) are masked by severe fluorescence. ${ }^{5}$ Thus, MTX is a typical example of a substance possessing strong SERS enhancement with simultaneous drastic fluorescence suppression. The latter is valuable since it may serve

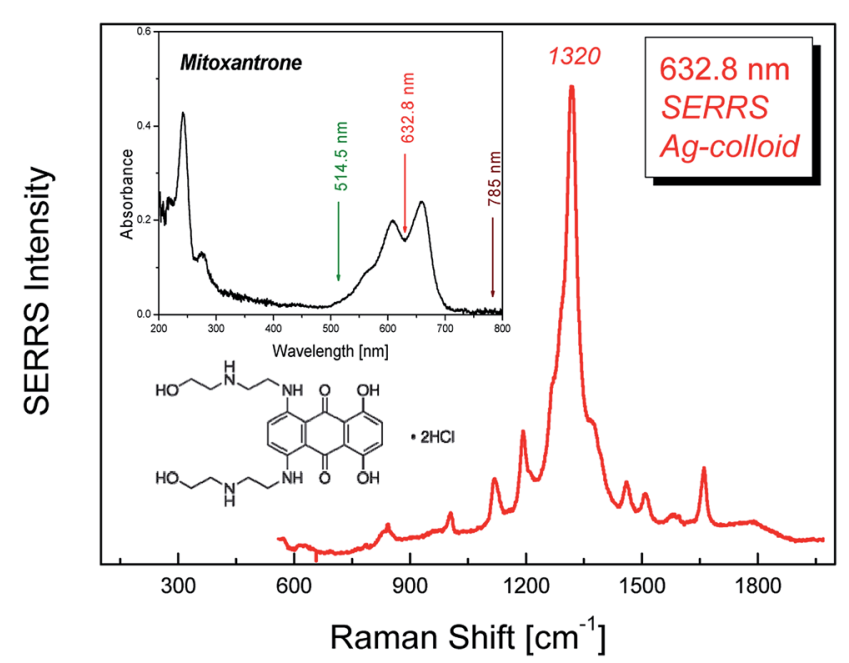

Fig. 1 Typical MTX SERRS spectrum using Ag-colloid substrate. Insets: absorption spectrum of MTX and its molecular structure. The three distinct laser wavelengths used in the study are marked with arrows. 
Table 1 Preparation parameters and morphological details of the substrates that contributed to the studies of the current work

\begin{tabular}{|c|c|c|c|c|c|c|}
\hline $\begin{array}{l}\text { Nominal } \\
\text { Au thickness [nm] }\end{array}$ & Sample name & $\begin{array}{l}\text { Annealing } \\
\text { temperature }\left[{ }^{\circ} \mathrm{C}\right]\end{array}$ & $\begin{array}{l}\text { Annealing } \\
\text { time [min] }\end{array}$ & $\begin{array}{l}\text { Particles } \\
\text { analyzed [Nb] }\end{array}$ & $\begin{array}{l}\text { Average size } \\
\left(d_{\text {av }}\right)[\mathrm{nm}]\end{array}$ & $\begin{array}{l}\text { Standard } \\
\text { deviation }[\mathrm{nm}]\end{array}$ \\
\hline 0 & $\mathrm{Si}$ & - & - & - & - & - \\
\hline$<1$ & Si_106 & 900 & 1 & 23480 & 6.5 & 2.1 \\
\hline 1 & Si_12 & 900 & 1 & 1626 & 23.8 & 8.3 \\
\hline 1 & Si_101 & 400 & 30 & 1514 & 24 & 9.4 \\
\hline 1 & Si_9 & 900 & 1 & 1607 & 24.8 & 9.1 \\
\hline 1 & Si_66 & 400 & 30 & 1895 & 25.8 & 10.4 \\
\hline 1 & Si_11 & 900 & 1 & 1030 & 26.1 & 9 \\
\hline 1 & Si_102 & 400 & 30 & 825 & 27.7 & 10.6 \\
\hline 1 & Si_67 & 400 & 30 & 851 & 41.5 & 14.2 \\
\hline 1 & Si_68 & 400 & 30 & 1447 & 28.8 & 11.7 \\
\hline 2 & Si_80 & 400 & 30 & 1388 & 104.1 & 31.4 \\
\hline 2 & Si_82 & 400 & 30 & 1332 & 105.7 & 30.9 \\
\hline 5 & Si_54 & 400 & 30 & 2192 & 108.8 & 35.4 \\
\hline 2 & Si_81 & 400 & 30 & 2185 & 125.4 & 38.9 \\
\hline 2 & Si_58 & 900 & 15 & 1068 & 263.3 & 79.8 \\
\hline 2 & Si_56 & 500 & 15 & 1104 & 265.5 & 75.2 \\
\hline 2 & Si_61 & 900 & 15 & 927 & 265.6 & 92.3 \\
\hline 2 & Si_57 & 700 & 15 & 933 & 283.3 & 86.8 \\
\hline
\end{tabular}

as an indication of plasmonic action whenever this particular wavelength is being used in the experiments.

\subsection{Morphology of the SERS substrates}

A number of $\mathrm{Au}$ thin films was prepared by application of various protocols and served as SERS substrates. The nominal $\mathrm{Au}$ layer thickness and the thermal treatment factors, temperature and duration, were the adjustable parameters (Table 1). Prior to annealing, the morphology of most Au sputtered films on Si surfaces consists of irregular shaped large particles with high degree of connectivity.

However, after the thermal treatment distinct Au islands evolved with spatial arrangements based upon Au film thickness and annealing conditions. Their morphology is revealed in the corresponding SEM images, representative of which are given in Fig. 2. The Au-island size distribution for each substrate was determined with the aid of appropriate image processing analysis (ImageJ). The derived histograms for selected cases are shown in Fig. 3. Table 1 summarizes also the average island size and width of the distribution derived for each sample. The average diameter of the Au-nanoparticles formed on each substrate is dictated by the dewetting thermodynamics, which in turn depend on the type of substrate (interfacial properties of the native $\mathrm{SiO}_{2}$ film on $\mathrm{Si}$ and the metal - Au in this case), the initial Au nominal thickness as well as the annealing conditions. It appears that for the particular case of Si wafers the selection of appropriate $\mathrm{Au}$ nominal thickness play a predominant role on the island morphology while there is also considerable effect of the annealing parameters (temperature/duration). In principle, the greater the values for nominal thickness, annealing temperature and duration, the larger the nanostructures
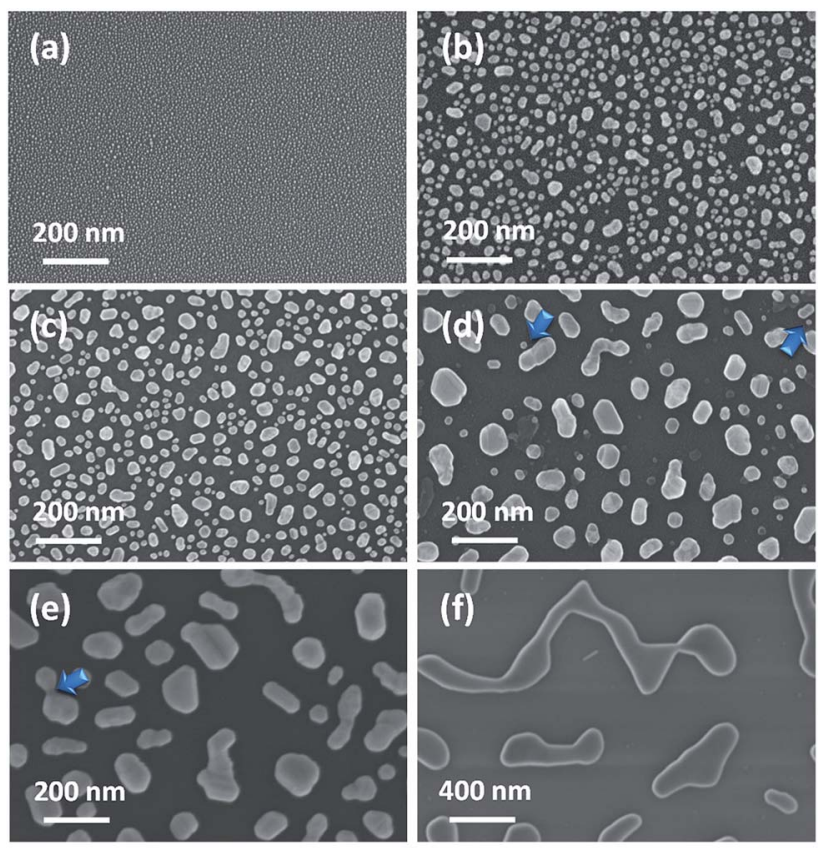

Fig. 2 SEM images of dewetted Au films. Initial nominal thickness and annealing parameters: (a) $<1 \mathrm{~nm}\left(900^{\circ} \mathrm{C}, 1 \mathrm{~min}\right),(\mathrm{b}) 1 \mathrm{~nm}\left(900{ }^{\circ} \mathrm{C}, 1\right.$ min), (c) $1 \mathrm{~nm}\left(400{ }^{\circ} \mathrm{C}, 30 \mathrm{~min}\right),\left(\right.$ d) $2 \mathrm{~nm}\left(400{ }^{\circ} \mathrm{C}, 30 \mathrm{~min}\right)$, (e) $5 \mathrm{~nm}$ $\left(400{ }^{\circ} \mathrm{C}, 30 \mathrm{~min}\right)$ and (f) $2 \mathrm{~nm}\left(900^{\circ} \mathrm{C}, 15 \mathrm{~min}\right)$. Arrows indicate possible dispersed hot spots for the substrates with $d_{\mathrm{av}}>30 \mathrm{~nm}$. 
(a)

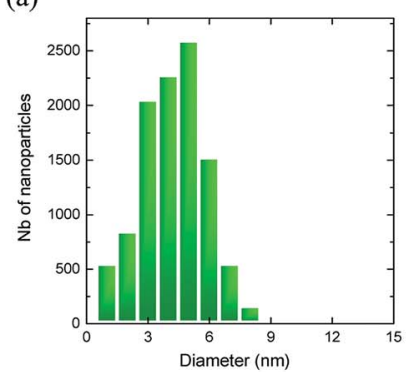

(c)

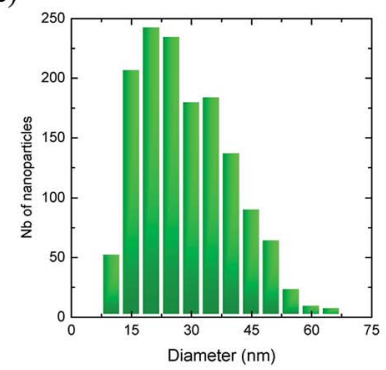

(e)

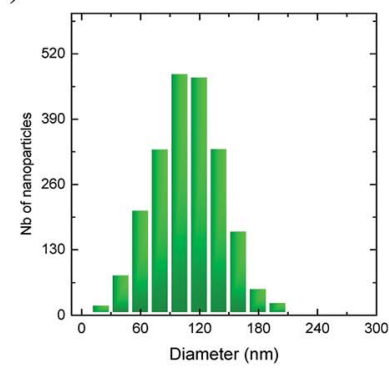

(b)

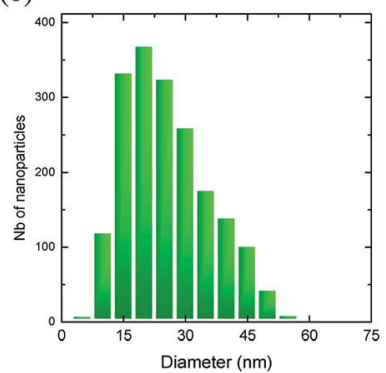

(d)

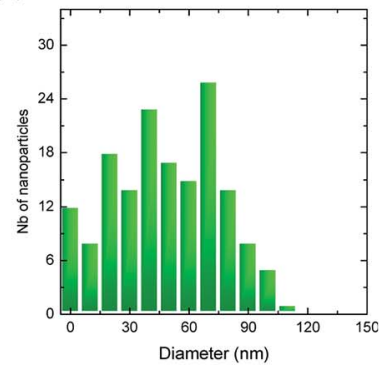

(f)



Fig. 3 Au island size distributions for the corresponding samples given in Fig. 2. The statistical analysis was performed on areas of $\sim 2 \mu \mathrm{m} \times 1.5$ $\mu \mathrm{m}$ for each sample.

formed. The aforementioned parameters enable the production of user-manipulated, reproducible substrates with well controlled particle size distributions ${ }^{21}$ (see also Table 1 ).

\subsection{Specular reflectance}

The performance of SERS is mainly dictated by the optical properties of the nanostructured substrates. The proposed substrates generate plasmon resonances which were probed via the respective UV-Vis spectra, representatively shown in Fig. 4(a).

The spectra are characterized by considerably broad bands in the visible spectral range that are red-shifted as the size of the $\mathrm{Au}$ nanostructures is increased. Correlation of the maximum of the associated absorption peak with respect to the Au-island average size is observed in Fig. 4(b) (error bars represent the full width at half maximum of the particle distribution function). The maxima of the plasmon resonant peaks, $\lambda_{\mathrm{p}}^{\max }$, span the $\sim 520$ to $650 \mathrm{~nm}$ wavelength range and exhibit an almost linear increase as a function of particle size (at least up to 60-70 $\mathrm{nm}$ ). It has been pointed out that the plasmon resonances of the islands strongly depend on their size and shape. Negative slopes of $\lambda_{\mathrm{p}}^{\max }$ as a function of island size have been reported and were

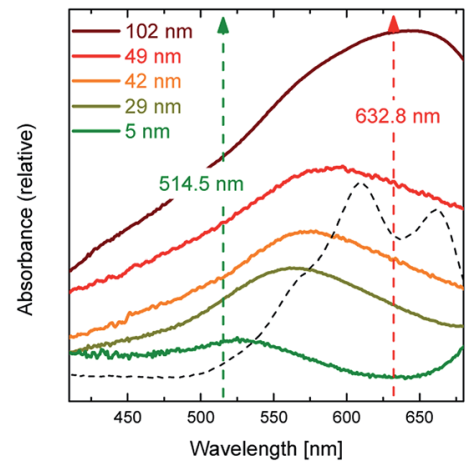

(a)

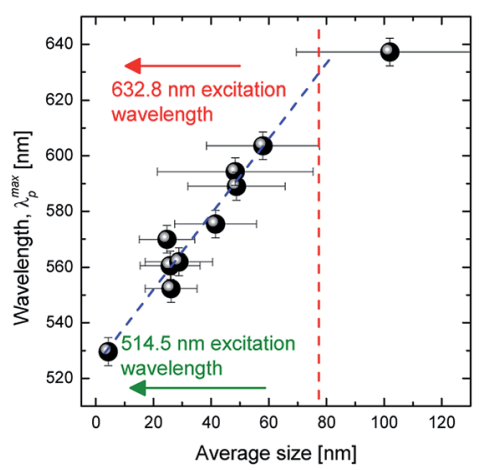

(b)

Fig. 4 (a) Absorbance spectra from representative substrates (different colours correspond to samples having different average Auisland size). The corresponding spectrum of an aqueous MTX solution (dashed line) is also added in order to reveal that for some of the substrates both resonance Raman and plasmon resonance conditions are fulfilled (when using $632.8 \mathrm{~nm}$ wavelength). (b) Correlation between the observed maxima of the plasmon resonant peaks (in the UV-Vis spectra) and the average size of Au-islands. Arrows denote the Raman/SERS excitation wavelengths while the dashed line indicates the average particle size of the Au islands that are expected to be in plasmon resonance with the $632.8 \mathrm{~nm}$ excitation wavelength.

explained by differences in particles' height in relation to their diameter. ${ }^{8}$ Our experimental findings (monotonic increase) suggest that the particle shape factor (aspect ratio) is roughly constant in all of the annealed samples. The differences found in the various studies may be explained by the physics associated with the dewetting processes i.e. the differences between the interactions in the metal-substrate interface. Fig. 4(b) indicates that the excitation wavelength $632.8 \mathrm{~nm}$ is evidently in plasmon resonance with the $\sim 80 \mathrm{~nm}$ in average Au-island size substrates, while the $514.5 \mathrm{~nm}$ excitation wavelength is in near plasmon resonance with the substrates having the particles of the smallest size, $\sim 5 \mathrm{~nm}$ (dashed line and arrows in Fig. 4).

\subsection{SERS-wavelength dependence}

The SERS substrates were studied with respect to the relative enhancement they offer on the scattering ability of MTX. Raman spectra were obtained from substrates having average Au-island size $\sim 30 \mathrm{~nm}$ upon which aqueous solutions of MTX of four different concentrations were spin coated. Three different excitation wavelengths (514.5, 632.8 and $785 \mathrm{~nm}$ ) were used in 


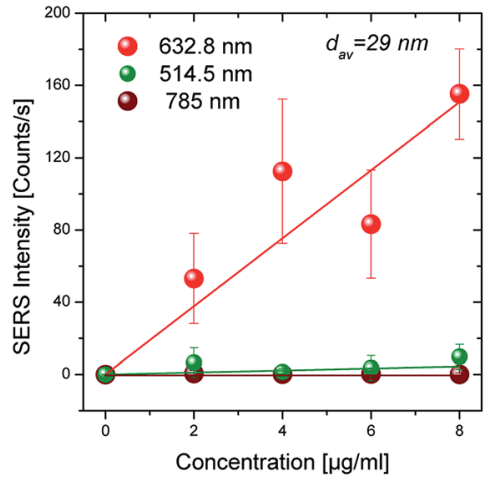

Fig. 5 SERS intensity for three different excitation wavelengths as a function of nominal concentration of the aqueous solutions that were spin coated on the $29 \mathrm{~nm}$ average Au-island size Si wafer (laser excitation power: $0.6 \mathrm{~mW}$, integration time: $30 \mathrm{~s}$ ).

order to check which of them offers the greatest enhancement. In order to minimize photo-induced phenomena, low excitation laser power on the sample was selected for all cases; these phenomena are more probable for the case of the $632.8 \mathrm{~nm}$ wavelength which falls in the absorption band of the MTX molecule (inset in Fig. 1). The results on the $29 \mathrm{~nm}$ average size Au-islands, given in Fig. 5, indicate a pronounced enhancement for the case of the $632.8 \mathrm{~nm}$ wavelength, with particularly weak enhancement for the $514.5 \mathrm{~nm}$. No signal was detected when using the $785 \mathrm{~nm}$ wavelength; for the latter case no signal was recorded for all concentrations used on any of the studied substrates; while for the particles with average diameter $<100 \mathrm{~nm}$ this may be explained by the lack of plasmon resonances, this does not apply for the case of particles with diameters $>100 \mathrm{~nm}$. In addition, extremely weak signals were recorded in the $514.5 \mathrm{~nm}$ case (almost two orders of magnitude lower than the ones observed when using the $632.8 \mathrm{~nm}$ ). Indeed, spectra collected even from the smallest $\mathrm{Au}$-island size ( $\sim 5 \mathrm{~nm}$ ), having plasmon resonances close to this particular wavelength $(514.5 \mathrm{~nm})$, were characterized by very low intensities. Contrary to the two aforementioned wavelengths exhibiting low or even no SERS activity, excitation with $632.8 \mathrm{~nm}$ enabled measurable intensities for all samples with $5<d_{\mathrm{av}}<$ $110 \mathrm{~nm}$, which are strongly dependent on both MTX concentration and the $\mathrm{Au}$ islands size.

The case of $d_{\mathrm{av}} \sim 30 \mathrm{~nm}$ is a characteristic one since particles with this diameter are in near plasmon resonance with both $514.5 \mathrm{~nm}$ and $632.8 \mathrm{~nm}$ excitation wavelengths. From the discussion above it becomes evident that the resonance Raman effect plays a decisive role on the intensity of the recorded Raman signal and should thus be considered as a significant contribution in addition to the enhancement offered by SERS. This is probably the reason (SERRS) that measurable intensities are detected for almost all samples when using the $632.8 \mathrm{~nm}$ excitation wavelength. For the same substrate with $d_{\mathrm{av}} \sim 30 \mathrm{~nm}$ size of the Au-islands, the intensity was found to vary from the position that the spectrum was obtained from; the error bars in Fig. 5 are the standard deviation of thirty measurements obtained within a sample's area of $\sim 30 \mathrm{~mm}^{2}$. Furthermore, despite the wide standard deviation, Fig. 5 indicates that the SERS intensity increases almost linearly with the nominal concentration of the MTX aqueous solution which is beneficial when attempting quantitative results.

\subsection{SERRS - the role of Au-island size}

The size of the Au islands critically affects plasmonic properties of the substrate and consequently the Raman scattering enhancement. The relative enhancement with respect to the different $\mathrm{Au}$ island average size was studied after deposition of the same concentration of MTX on each substrate. For the evaluation of the island size effect the most favorable excitation wavelength, for which SERRS conditions are fulfilled i.e. $632.8 \mathrm{~nm}$, was selected. The results for the case of $1.5 \mu \mathrm{g} \mathrm{ml}^{-1}$ are shown in Fig. 6 and indicate the manifestation of a maximum in the SERRS intensity at the average Au island size of $\sim 70-80 \mathrm{~nm}$. This observation is in accordance with the corresponding island size with a plasmon resonance wavelength $\sim 632.8 \mathrm{~nm}$ (dashed line in Fig. 4(b)). The SERRS intensity is greatly diminished when the size of the nanodroplets falls below $\sim 40 \mathrm{~nm}$ or exceeds the value of $\sim 110 \mathrm{~nm}$. Apart from $\mathrm{Au}$ island size, the distance between particles plays an additional role. Liu et al. ${ }^{22}$ have evaluated the inter-particle contribution to the SERS intensity for a nanopost array.

They stressed that the enhancement is achieved mainly from the contribution of the edge of the posts and the coupling effect, but the enhancement from the top of the post cannot be ignored. Their calculations along with experimental evidence indicated an increase in the normalized SERS intensity (normalized with respect to the number of SERS sites per scattering volume) by a factor of two when the $100 \mathrm{~nm}$ sized islands are separated by a distance of $d \sim 50 \mathrm{~nm}$ with

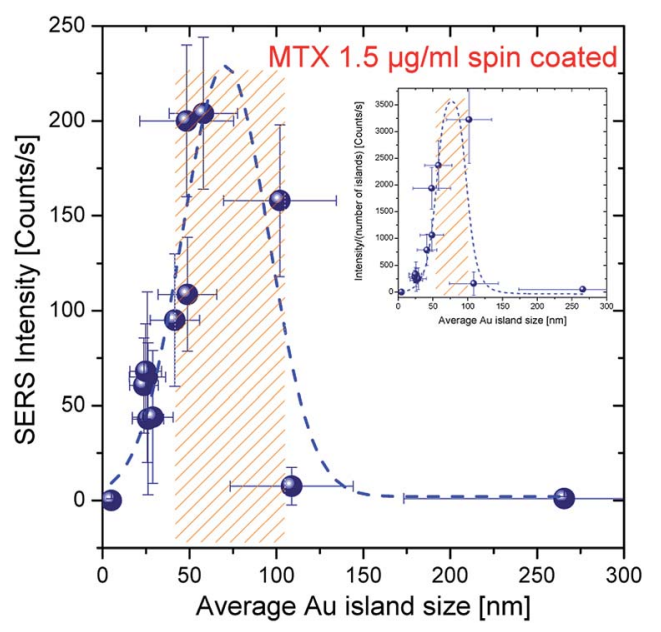

Fig. 6 Size dependence of SERS intensity for the case of $1.5 \mu \mathrm{g} \mathrm{ml}^{-1}$ MTX droplet concentration (dashed line is drawn as a guide to the eye; shadowed region indicates the island sizes in average that offer at least $50 \%$ of the maximum SERRS intensity). The corresponding normalized SERS intensity (according to ref. 22) is plotted in the inset (laser excitation power: $0.6 \mathrm{~mW}$, integration time: $30 \mathrm{~s}$ ). 
respect to the intensity observed for longer inter-particle distances $(d>200 \mathrm{~nm})$. Even though experimental data were not collected for $d<50 \mathrm{~nm}$ the general trend of the data presented in their work as well as the well-established "hot spot" generation for such low inter-particle distances point toward even higher enhancement factors. Visual inspection of the SEM images reveal that the interparticle distance of Au particles becomes less than $\sim 50 \mathrm{~nm}$ mainly for particles with low average diameter, i.e. $<\sim 30 \mathrm{~nm}$, see for example Fig. 2(a)-(c). For substrates with $d_{\mathrm{av}}>30 \mathrm{~nm}$, the distance between $\mathrm{Au}$ islands is larger than $50 \mathrm{~nm}$ and therefore this parameter is not expected to play significant role in the overall enhancement factor. However, in accordance with ref. 7 there may statistically exist sites where the inter-particle distance is $<50 \mathrm{~nm}$ (indicated by arrows in Fig. 2(d) and (e)).

The inset in Fig. 6 shows the normalized SERS intensity as a function of average $\mathrm{Au}$ island size (with respect to the number of islands within the scattering volume) and indicates a maximum at $d_{\mathrm{av}} \sim 80 \mathrm{~nm}$ in substrates with only a few hot spot sites.

\subsection{SERRS - semi-quantitative measurements}

The semi-quantitative measurement of molecular species (MTX in the current work) offered by the proposed SERS substrates is revealed by the linear dependence of the SERRS signal recorded on the nominal concentration of the species spread on them. Fig. 7 depicts this behavior in the range of $0-$ $2 \mu \mathrm{g} \mathrm{ml} \mathrm{m}^{-1}$ concentration spread on four substrates with different $d_{\text {av }}$. An almost linear behavior has already been shown to persist roughly up to the concentrations of $10 \mu \mathrm{g}$ $\mathrm{ml}^{-1} \cdot{ }^{21}$ Considerably large error bars associated with the measurements may be explained by the small ensemble of Au-islands sampled when using the $50 \times$ objective. The absolute quantity of MTX that is actually deposited on the plasmonic surface after the respective droplet has been spin coated on the substrate will allow quantitative measurements to be attained.

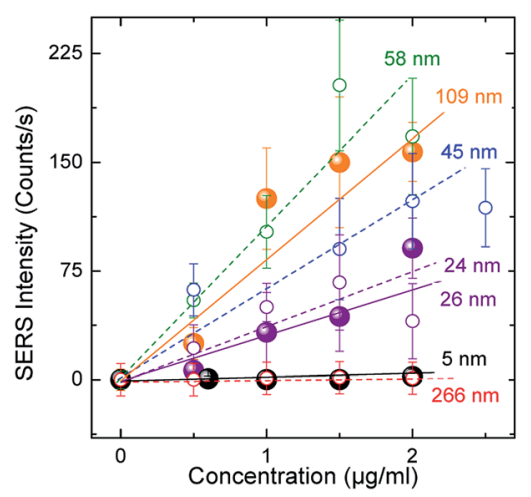

Fig. 7 The dependence of SERS intensity on concentration for substrates possessing different $\mathrm{Au}$ island diameters (laser excitation power: $0.6 \mathrm{~mW}$, integration time: $30 \mathrm{~s}$ ). Dashed/solid lines represent linear fits to the corresponding "open"/"solid" data points of the same colour.

\subsection{SERRS - quantitative measurements}

Quantitative measurements can be accomplished only if the actual quantity of MTX spread on the plasmonic surfaces after the spin-coating process is estimated. Based on the experience to routinely apply SERS colloidal substrates for the quantification of MTX at extremely low concentrations, in the order of pg $\mathrm{ml}^{-1}, 2$ we applied the following methodology (Fig. 8) for the quantification of MTX which is essentially spread on the Auisland plasmonic surfaces. The substrate (e.g. the one for which $2 \mu \mathrm{g} \mathrm{ml}^{-1}$ solution was spin coated on the Si wafer) was carefully cleaned at its edges (under a stereo-microscope) in order to remove the concentrated MTX quantities formed during the spinning process and was subsequently immersed in $2 \mathrm{ml}$ of triple distilled water which was sonicated for $15 \mathrm{~min}$. Quantification of the extracted molecular species involves SERS experiments from Ag-colloidal substrates prepared using the Lee \& Meisel method ${ }^{23}$ according to ref. 2. In brief, quantity of $100 \mu \mathrm{l}$ from the extracted solution was transferred in a pyrextube containing $1 \mathrm{ml}$ of Ag-colloidal SERS substrate and quantification of MTX was attained by recording the intensity of the $90^{\circ}$ scattering geometry SERS signal offered from the colloidal substrate. This intensity was corresponded to the concentration of MTX in the $2 \mathrm{ml}$ solution through an appropriate calibration curve which was built for this particular colloidal substrate using reference MTX aqueous solutions. In this manner, the unknown concentration of MTX in the $2 \mathrm{ml}$ solution extracted after the sonication was found to be $\sim 50 \mathrm{pg} \mathrm{ml}^{-1}$; indicating that the estimated total mass of MTX on the silicon wafer was $\sim 0.2 \mathrm{pM} \mathrm{cm}^{-2}$. The latter corresponds to a number of MTX molecules of less than $\sim 10^{3}$ per scattering volume (spot of the focused laser beam on sample $\sim 2 \mu \mathrm{m}^{2}$ ) making it less than a few hundreds of molecules for the lower concentrations of the solutions that were spin coated on the active substrates. The enhancement factor is particularly difficult to be extracted since the conventional Raman signal of MTX cannot be obtained under the same experimental conditions as the ones used for the SERS experiments. To resolve this issue Raman spectra of reference solid and aqueous MTX solutions obtained in off resonance conditions (441.6 $\mathrm{nm}$ ) were taken into account. According to Le $\mathrm{Ru}$ et $a .^{24}$ the enhancement factor can be extracted from the equation:



Fig. 8 Schematic figure of the steps followed according to the proposed methodology for the quantification of MTX that is spread on the substrates. 


$$
\mathrm{EF}=\frac{I_{\text {SERRS }} / N_{\text {Surf }}}{I_{\mathrm{RS}} / N_{\mathrm{Vol}}}
$$

where EF is the overall enhancement factor (SERS in addition to resonance), $N_{\mathrm{Vol}}=C_{\mathrm{RS}} V$ is the average number of molecules in the scattering volume for the Raman (non SERS, non resonance) measurement and $N_{\text {Surf }}$ is the average number of adsorbed molecules in the scattering volume for the SERRS experiments. For the case of $2 \mu \mathrm{g} \mathrm{ml}^{-1}$ solution spin coated on the substrates, $N_{\text {Surf }}$ was experimentally approached as $\sim 1000$ molecules. $I_{\mathrm{RS}}$ is the conventional Raman intensity which for verification purposes was obtained for two separate cases: (a) for a MTX aqueous solution of known concentration $C_{\mathrm{RS}}=0.510^{-3} \mathrm{~g} \mathrm{ml}^{-1}$ where $I_{\mathrm{RS}} \sim 1$ count per s and (b) for a crystalline MTX sample of known density $\left(\sim 1.5 \mathrm{~g} \mathrm{~cm}^{-3}\right)$ where $I_{\mathrm{RS}} \sim 1000$ counts per $\mathrm{s}$. The overall enhancement factor offered when in both plasmon and resonance Raman conditions was estimated using eqn (1) in the order of $10^{9}$. Under the hypothesis that: (a) all MTX molecules are adsorbed on the $\mathrm{Au}$ islands and (b) the probed volume $\mathrm{V}$ (for the specific microscope objective used $50 \times$, NA 0.55 ) is estimated to be $>10^{4} \mu \mathrm{m}^{3}$, the extracted EF is actually a lower limit value.

Given that the methodology followed for the production of the particular substrates does not generate great numbers of "hot spots", the estimated enhancement factor is considered as particularly high. Control of the number of hot spots in more elaborate samples may allow even greater enhancement factors.

\section{Conclusions}

The enhancement of the scattered intensity associated with a prototype molecule (MTX) due to plasmon resonances of properly designed SERS substrates was thoroughly studied. Several parameters such as excitation wavelength, nominal thickness and annealing parameters are considered and optimization processes are proposed that may be applied, consistent with the molecular species that require identification/ quantification. These processes take advantage of both the local field enhancement and the resonance Raman effect and determine the structural properties of the substrates that ascertain the lowest detection limits. It is shown that SERRS intensity possesses a maximum as a function of Au-island average size and for the specific case of MTX this size is $\sim 80 \mathrm{~nm}$. The substrates proposed in the work were tested with respect to the semi-quantitative results they offer and linear relations of the scattered intensity as a function of the quantity of the detected species were found. A method that deals with the problem of identification and quantification (absolute values) is also suggested. The simplicity and repeatability of the optimization process proposed compensates for the more elaborate substrates that target the generation of a great number of hot spots.

\section{Conflicts of interest}

There are no conflicts to declare.

\section{Acknowledgements}

We acknowledge support of this work (a) by the EC project NanoBarrier (FP7/2012-2016 under grant agreement no. 280759), (b) by the project "Materials and Processes for Energy and Environment Applications" (MIS 5002556) implemented under the "Action for the Strategic Development on the Research and Technological Sector", funded by the Operational Programme "Competitiveness, Entrepreneurship and Innovation" (NSRF 2014-2020) and co-financed by Greece and the European Union (European Regional Development Fund) and (c) by the Stavros Niarchos Foundation within the framework of the project ARCHERS ("Advancing Young Researchers' Human Capital in Cutting Edge Technologies in the Preservation of Cultural Heritage and the Tackling of Societal Challenges”).

\section{Notes and references}

$1 \mathrm{~S}$. Schlucker, Surface Enhanced Raman Spectroscopy: analytical, biophysical and life science applications, WileyVCH, Weinheim, Germany, 2011.

2 J. A. Anastasopoulos, A. Soto-Beobide and G. A. Voyiatzis, J. Raman Spectrosc., 2013, 44, 401.

3 E. Kämmer, K. Olschewski, T. Bocklitz, P. Röschet, K. Weber, D. Cialla and J. Popp, Phys. Chem. Chem. Phys., 2014, 16, 9056.

4 K. R. Strehle, D. Cialla, P. Rösch, T. Henkel, M. Köhler and J. Popp, Anal. Chem., 2007, 79, 1542.

5 A. C. Manikas, A. Soto-Beobide and G. A. Voyiatzis, Analyst, 2009, 134, 587.

6 V. L. Schlegel and T. M. Cotton, Anal. Chem., 1991, 63, 241.

7 P. Pavaskar, I.-K. Hsu, J. Theiss, W. H. Hung and S. B. Cronin, J. Appl. Phys., 2013, 113, 034302.

8 R. P. Van Duyne, J. C. Hulteen and D. A. Treichel, J. Chem. Phys., 99, 2101.

9 G. D. Sockalingum, A. Beljebbar, H. Morjani, J. F. Angiboust and M. Manfait, Biospectroscopy, 1998, 4, S71.

10 G. Santoro, S. Yu, M. Schwartzkopf, P. Zhang, S. Koyiloth Vayalil, J. F. H. Risch, M. A. Rübhausen, M. Hernández, C. Domingo and S. V. Roth, Appl. Phys. Lett., 2014, 104, 243107.

11 G. Q. Liu, Z. Q. Liu, Y. H. Chen, K. Huang, L. Li, F. L. Tang, L. X. Gong, Y. Hu and X. N. Zhang, Optik, 2013, 124, 5124.

12 M. Suzuki, Y. Niidome and S. Yamada, Colloids Surf., A, 2006, 284-285, 388.

13 P.-P. Fang, J.-F. Li, Z.-L. Yang, L.-M. Li, B. Ren and Z.-Q. Tian, J. Raman Spectrosc., 2008, 39, 1679.

14 J. Quan, J. Zhang, X. Qi, J. Li, N. Wang and Y. Zhu, Sci. Rep., 2017, 7, 14771.

15 E. Solary, D. Caillot, B. Chauffert, R.-O. Casasnovas, M. Dumas, M. Maynadie and H. Guy, J. Clin. Oncol., 1992, 10, 1730.

16 J. A. Neidhart, D. Gochnour, R. Roach, D. Hoth and D. Young, J. Clin. Oncol., 1986, 4, 672.

17 L. Salvagno, A. Contu, A. Bianco, L. Endrizzi, G. M. Schintu, N. Olmeo, S. M. L. Aversa, V. Chiarion-Sileni, M. Sorarù and M. V. Fiorentino, Ann. Oncol., 1992, 3, 833. 
18 F. Lawton, G. Blackledge, J. Mould, T. Latief, R. Watson and A. D. Chetiyawardana, Cancer Treat. Rep., 1987, 71, 627.

19 N. Strekal, A. Maskevich, S. Maskevich, J.-C. Jardillier and I. Nabiev, Biopolymers, 2000, 57, 325.

20 K. Govatsi, A. Chrissanthopoulos, V. Dracopoulos and S. N. Yannopoulos, Nanotechnology, 2014, 25, 215601.
21 S. Andrikaki, K. Govatsi, S. N. Yannopoulos, G. A. Voyiatzis and K. S. Andrikopoulos, Adv. Device Mater., 2017, 3, 30.

22 Y.-J. Liu, Z.-Y. Zhang, Q. Zhao and Y.-P. Zhao, Appl. Phys. Lett., 2008, 93, 173106.

23 C. Lee and D. Meisel, J. Phys. Chem., 1982, 86, 3391.

24 E. C. Le Ru, E. Blackie, M. Meyer and P. G. Etchegoin, J. Phys. Chem. C, 2007, 111, 13794. 